\title{
A MEASUREMENT OF THE ANGULAR POWER SPECTRUM OF THE CMB TEMPERATURE ANISOTROPY FROM THE 2003 FLIGHT OF BOOMERANG
}

\author{
W. C. Jones, ${ }^{1}$ P. A. R. Ade, ${ }^{2}$ J. J. Bock,${ }^{3}$ J. R. Bond,${ }^{4}$ J. Borrill,${ }^{5,6}$ A. Boscaleri, ${ }^{7}$ P. Cabella, ${ }^{8}$ C. R. Contaldi, ${ }^{4,9}$ \\ B. P. Crilll ${ }^{10}$ P. de Bernardis, ${ }^{11}$ G. De Gasperis, ${ }^{8}$ A. de Oliveira-Costa, ${ }^{12}$ G. De Troia, ${ }^{11}$ G. di Stefano, ${ }^{13}$ \\ E. Hivon, ${ }^{10}$ A. H. Jaffe, ${ }^{9}$ T. S. Kisner, ${ }^{14,15}$ A. E. Lange, ${ }^{1}$ C. J. MacTavish, ${ }^{16}$ S. Masi, ${ }^{11}$ P. D. Mauskopf ${ }^{2}$ \\ A. Melchiorri, ${ }^{11,17}$ T. E. Montroy, ${ }^{15}$ P. Natoli, ${ }^{8,18}$ C. B. Netterfield, ${ }^{16,19}$ E. Pascale, ${ }^{16}$ F. Piacentini, ${ }^{11}$ \\ D. Pogosyan, ${ }^{4,20}$ G. Polenta, ${ }^{11}$ S. Prunet, ${ }^{21}$ S. Ricciardi, ${ }^{11}$ G. Romeo, ${ }^{13}$ J. E. Ruhl, ${ }^{15}$ \\ P. Santini, ${ }^{11}$ M. Tegmark, ${ }^{12}$ M. Veneziani, ${ }^{11}$ and N. Vittorio ${ }^{8,18}$ \\ Received 2005 July 21; accepted 2006 April 28
}

\begin{abstract}
We report on observations of the cosmic microwave background (CMB) obtained during the 2003 January flight of BOOMERANG. These results are derived from $195 \mathrm{hr}$ of observation with four $145 \mathrm{GHz}$ polarization-sensitive bolometer (PSB) pairs, identical in design to the four $143 \mathrm{GHz}$ Planck High Frequency Instrument (HFI) polarized pixels. The data include $75 \mathrm{hr}$ of observations distributed over $1.84 \%$ of the sky with an additional $120 \mathrm{hr}$ concentrated on the central portion of the field, which represents $0.22 \%$ of the full sky. From these data we derive an estimate of the angular power spectrum of temperature fluctuations of the CMB in 24 bands over the multipole range $50 \leq$ $l \leq 1500$. A series of features, consistent with those expected from acoustic oscillations in the primordial photonbaryon fluid, are clearly evident in the power spectrum, as is the exponential damping of power on scales smaller than the photon mean free path at the epoch of last scattering $(l \gtrsim 900)$. As a consistency check, the collaboration has performed two fully independent analyses of the time-ordered data, which are found to be in excellent agreement.
\end{abstract} Subject headings: cosmic microwave background - cosmology: observations - instrumentation: detectors Online material: color figures

\section{INTRODUCTION}

The wealth of cosmological information that is encoded in the statistical properties of the cosmic microwave background radiation (CMB) has motivated a highly successful observational effort to measure the angular power spectrum of the CMB temperature anisotropies. The experimental effort is broad-based, with teams reporting results from interferometric and single-dish observations spanning a decade in frequency and more than three decades in angular scale (for some recent results, see Bennett et al.

\footnotetext{
1 Physics Department, California Institute of Technology, MS 59-33, 1200 East California Boulevard, Pasadena, CA 91125; wcj@astro.caltech.edu.

2 School of Physics and Astronomy, Cardiff University, Cardiff CF24 3YB, UK.

3 Jet Propulsion Laboratory, Pasadena, CA 91109.

${ }^{4}$ Canadian Institute for Theoretical Astrophysics (CITA), University of Toronto, Toronto, ON M5S 3H8, Canada.

5 Computational Research Division, Lawrence Berkeley National Laboratory, Berkeley, CA 94720.

6 Space Sciences Laboratory, University of California, Berkeley, CA 94720.

7 IFAC-CNR, 50127 Florence, Italy.

8 Dipartimento di Fisica, Università di Roma "Tor Vergata," 00133 Rome, Italy.

9 Theoretical Physics Group, Imperial College, London SW7 2BW, UK.

${ }^{10}$ IPAC, California Institute of Technology, Pasadena, CA 91125.

11 Dipartimento di Fisica, Università di Roma "La Sapienza," 00185 Rome, Italy.

12 Department of Physics, Massachusetts Institute of Technology, Cambridge, MA 02139.

13 Instituto Nazionale di Geofisica e Vulcanologia, 00143 Rome, Italy.

14 Department of Physics, University of California, Santa Barbara, CA 93106.

15 Department of Physics, Case Western Reserve University, Cleveland, $\mathrm{OH}$ 44106.

16 Department of Physics, University of Toronto, Toronto, ON M5S 3H8, Canada.

17 INFN, Sezione di Roma 1, 00133 Rome, Italy.

18 INFN, Sezione di Roma 2, 00133 Rome, Italy.

19 Department of Astronomy and Astrophysics, University of Toronto, Toronto, ON M5S 3H8, Canada.

20 Department of Physics, University of Alberta, Edmonton, AB T6G 2J1, Canada.

${ }^{21}$ Institut d'Astrophysique de Paris, 75014 Paris, France.
}

2003b; Tristram et al. 2005b; Ruhl et al. 2003; Halverson et al. 2002; Readhead et al. 2004; Kuo et al. 2004; Dickinson et al. 2004; Lee et al. 2001). The success of these observations, coupled with the predictive power of accurate theoretical modeling, has contributed to the ongoing transformation of cosmology into a quantitative, precise, and increasingly accurate science.

In this paper we present the angular power spectrum of the temperature anisotropies derived from the data obtained during the 2003 January flight of BOOMERANG (hereafter B03). Having been upgraded with a polarization-sensitive receiver (Montroy et al. 2003; Masi et al. 2006), B03 is optimized to probe the polarization of the CMB at subdegree angular scales while retaining full sensitivity to the unpolarized emission. In addition to a measurement of the curl-free component of the CMB polarization (Montroy et al. 2006) and the temperature-polarization crosscorrelation (Piacentini et al. 2006), the 2003 flight has resulted in a precise measurement of the angular power spectrum of the temperature anisotropies. These data represent an improvement on published measurements of the temperature power spectrum at multipoles $600 \lesssim l \lesssim 1200$, which probe physical scales corresponding to the photon mean free path during the epoch of last scattering.

\section{INSTRUMENT AND OBSERVATIONS}

Following the successful 1998 Antarctic campaign (de Bernardis et al. 2000; Netterfield et al. 2002; Ruhl et al. 2003; Crill et al. 2003), an entirely new focal plane was designed around a set of four $145 \mathrm{GHz}$ PSB pairs. These receivers, the first of their kind to be used in astronomical observations, combine the raw sensitivity of cryogenic bolometers with intrinsic sensitivity to linear polarization, a property historically associated only with coherent detectors (Jones 2005; Jones et al. 2003; Montroy 2003).

In addition to the four $145 \mathrm{GHz}$ PSB pixels, the B03 focal plane accommodates four dual-frequency photometers, operating in bands centered near 245 and $345 \mathrm{GHz}$. While the CMB 
TABLE 1

B03 Instrument Summary

\begin{tabular}{|c|c|c|c|c|c|}
\hline $\begin{array}{c}\langle\nu\rangle \\
(\mathrm{GHz})\end{array}$ & $\begin{array}{l}\text { MJy sr }{ }^{-1} \\
\text { Per } \mathrm{K}_{\mathrm{CMB}}\end{array}$ & $\begin{array}{l}\theta_{\text {phys }} \text { FWHM } \\
\quad(\operatorname{arcmin})\end{array}$ & $\begin{array}{c}\theta_{\mathrm{eff}^{\mathrm{a}}} \text { FWHM } \\
(\operatorname{arcmin})\end{array}$ & $\begin{array}{c}\mathrm{NET}^{\mathrm{b}} \\
\left(\mu \mathrm{K}_{\mathrm{CMB}} \mathrm{s}^{1 / 2}\right)\end{array}$ & $\begin{array}{c}\sigma_{\mathrm{pix}}{ }^{\mathrm{c}} \\
\left(\mu \mathrm{K}_{\mathrm{CMB}}\right)\end{array}$ \\
\hline ................ & 388 & 9.95 & 11.5 & 63 & 24 \\
\hline $245 \ldots \ldots \ldots \ldots \ldots \ldots \ldots \ldots \ldots$ & 462 & 6.22 & 8.5 & 161 & 62 \\
\hline $345 \ldots \ldots \ldots \ldots \ldots \ldots \ldots \ldots$ & 322 & 6.90 & 9.1 & 233 & 89 \\
\hline
\end{tabular}

\footnotetext{
a The effective beam is defined as the convolution of the physical beam with the $\simeq 2$ '. 4 rms error in the pointing reconstruction as determined from point-source observations in the CMB field.

$\mathrm{b}$ The focal plane noise-equivalent temperature, derived from the in-flight noise measured at $1 \mathrm{~Hz}$. Note, however, that at both low $(<100 \mathrm{mHz})$ and high $(>5 \mathrm{~Hz})$ frequencies, the noise rises significantly. The focal plane accommodates eight detectors at $145 \mathrm{GHz}$ and four detectors at each of 245 and $345 \mathrm{GHz}$.

c The approximate noise $\left[\approx\left\langle\operatorname{diag}\left(\boldsymbol{C}_{N}\right)\right\rangle^{1 / 2}\right]$ per 3.'4 pixel in the deep field for the data included in this analysis.
}

temperature anisotropies are detected with high signal-to-noise ratio in the 245 and $345 \mathrm{GHz}$ data (Montroy 2003; Masi et al. 2006), these data are not included in the present analysis. Further study is required to understand the detailed properties of the noise and the polarization systematics for these channels.

\subsection{Polarization Sensitive Bolometers}

Each bolometer within a PSB pair is sensitive to a linear combination of the Stokes $I, Q$, and $U$ parameters on the sky multiplied by the frequency response of the receiver, $F_{\nu}$, and convolved with the (two-dimensional) co- and cross-polar beam patterns, $P_{\|}(\hat{\boldsymbol{r}})$ and $P_{\perp}(\hat{\boldsymbol{r}})$, respectively. After deconvolving the system transfer function from the time-ordered data (TOD), each sample from a single detector within a PSB pair, $d_{i}$, may be written as the sum of a signal component,

$$
\begin{aligned}
d_{i}= & \frac{s}{2} \int d \nu \frac{\lambda^{2}}{\Omega_{b}} F_{\nu} \iint d \hat{\boldsymbol{r}}\left[P_{\|}\left(\hat{\boldsymbol{r}}_{i}\right)+P_{\perp}\left(\hat{\boldsymbol{r}}_{i}\right)\right] \\
& \times\left[I+\gamma \mathcal{P}\left(\hat{\boldsymbol{r}}_{i}\right)\left(Q \cos 2 \psi_{i}+U \sin 2 \psi_{i}\right)\right],
\end{aligned}
$$

and a noise contribution. The Stokes parameters are understood to be defined on the full sky, and the integration variable is $\hat{\boldsymbol{r}}_{i}=$ $\hat{\boldsymbol{n}}_{i}-\hat{\boldsymbol{r}}$, for a vector, $\hat{\boldsymbol{n}}_{i}$, describing the pointing at a time sample, $i$. The normalized beam response and the polarization efficiency are given by

$$
\mathcal{P}(\hat{\boldsymbol{r}}) \equiv \frac{P_{\|}-P_{\perp}}{P_{\|}+P_{\perp}}, \quad \gamma \equiv \frac{1-\epsilon}{1+\epsilon} .
$$

The polarization leakage parameter, $\epsilon$, is defined as the ratio of the square of the diagonal elements of the Jones matrix describing an imperfect polarizer. For B03's PSBs the leakage ranges from $5 \%$ to $8 \%$, as determined from preflight measurements. The angle $\psi$ is the projection of the axis of sensitivity of a given detector on the sky. This angle is modulated in time due to sky rotation and is further affected by the motion of the gondola. The calibration factor, $s$, that converts the brightness fluctuations in $I, Q$, and $U$ to a signal voltage is obtained through cross calibration with the temperature anisotropies observed by the Wilkinson Microwave Anistropy Probe (WMAP) (Bennett et al. 2003a; Masi et al. 2006).

A more detailed discussion of the PSB design may be found in Jones et al. (2003). A description of the characterization of the B03 PSBs may be found in Masi et al. (2006). A discussion regarding properties and methods of analysis of PSBs may be found in Jones et al. (2006) and Jones (2005).

\subsection{Sky Coverage}

The BOOMERANG gondola scans in azimuth, mapping the sky signal to a bandwidth between $\sim 50 \mathrm{mHz}$ and $5 \mathrm{~Hz}$. Sky rotation modulates the orientation of the instrument with respect to the signal. A complete characterization of the Stokes $I, Q$, and $U$ parameters is achieved through a combination of sky rotation and the joint analysis of the data from all eight detectors during the course of pixelization. The low-frequency stability of the bolometric system and balloon environment allow an accurate reconstruction of all three linear Stokes parameters at each pixel with sensitivity to angular scales ranging from $\sim 10^{\circ}$ to the $\sim 10^{\prime}$ beam size.

The launch date, latitude, and the azimuth/elevation constraints imposed by solar, limb, and balloon avoidance considerations limit the region of sky accessible to an Antarctic long-duration balloon (LDB) payload. During the austral summer, the antisolar meridian falls in the vicinity of R.A. $\simeq 70^{\circ}$ (J2000.0 epoch). Continuum emission from the Galaxy is significant over much of the available sky; the CMB field is chosen to minimize this Galactic contamination, subject to the constraints imposed by the LDB flight parameters.

The BOOMERANG telescope is an altitude-azimuth mount that is scanned in azimuth at constant elevation. The elevation is adjusted on hour timescales. The pendulation frequencies of the gondola and scan rate limitations constrain the minimum peak to peak scan amplitude to approximately $15^{\circ}$. Earth's rotation provides about $20^{\circ}$ of cross linking between scans, which aides in the decorrelation of the Stokes parameters. When the local hour angle (modulo $12 \mathrm{hr}$ ) is in the vicinity of the right ascension of the target field (i.e., when the sky rotation and therefore the cross linking is minimal), our scans are redirected to the Galactic plane.

Given B03's instantaneous sensitivity (shown in Table 1), the amplitude of the CMB polarization signal motivates us to concentrate as much integration time as possible in as small a region as possible. However, the competing desire to make high-fidelity measurements of (the relatively large amplitude) unpolarized temperature fluctuations led us to a two-tiered scan strategy, representing a compromise between sensitivity to the temperature and polarization power spectra.

During the first 4 days of the flight, $75 \mathrm{hr}$ were dedicated to observations covering $\simeq 1.84 \%$ of the sky (these observations are referred to as the "shallow field"), while an additional $120 \mathrm{hr}$ of observation were concentrated on the central portion of this field (the "deep region"). The deep field constitutes $\simeq 0.22 \%$ of the full sky. The exact distributions of integration time for the data included in this analysis are shown in Figure 1. The B03 sky coverage is a subset of the region observed during BOOMERANG's 1998 flight. 

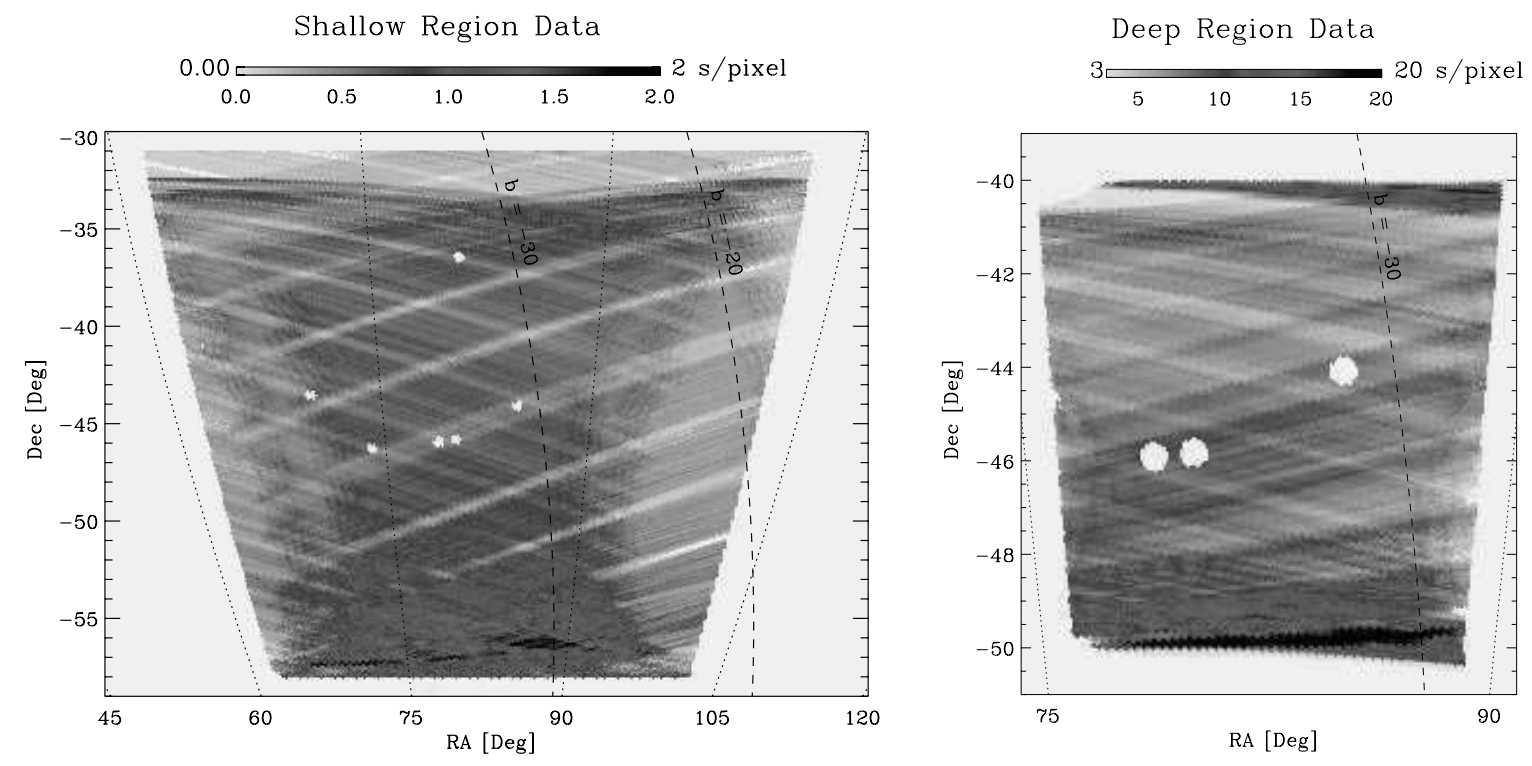

FIG. 1.-Sky coverage obtained during the 2003 January flight of BOOMERANG. The integration times per 3.'4 pixel are shown for the shallow and deep data subsets described in the text. Galactic latitude contours of $b=(-30,-20)$ are shown for reference. Approximately $75 \mathrm{hr}$ of observation are devoted to the shallow region, with an additional $120 \mathrm{hr}$ concentrated in the central deep field. The cross-linking of the scans due to sky rotation is evident in the diagonal striping of the coverage, which has a $\approx 20^{\circ}$ opening angle. Known extragalactic point sources, shown masked in these plots, are excluded from the analysis. [See the electronic edition of the Journal for a color version of this figure.]

Of the $257 \mathrm{hr}$ that are spent in observations of the $\mathrm{CMB}$ region, $195 \mathrm{hr}(76 \%)$ survived cuts based on pointing considerations. Of these data $6 \%$ are contaminated by small cosmic-ray hits, $0.5 \%$ by cosmic-ray hits that saturate the analog-to-digital converter in one or more channels, $1.5 \%$ from elevation changes, and $1.3 \%$ due to calibration lamp pulses. The total observing efficiency is therefore approximately $67 \%$, with the bulk of the cuts resulting from problems related to the pointing. After filling the flagged portions of the bolometer time streams with a realization of the noise, a fast Fourier transform (FFT) deconvolution of the entire time stream ( $\sim 12$ days) of each detector is performed in a single operation.

\subsection{In-Flight Calibration}

Both the calibration of the receivers and the characterization of the effective beam must be obtained from the in-flight data. The former is due to the dependence of the bolometer responsivity on the radiative background, while the latter depends on the fidelity of the pointing reconstruction.

The calibrations are obtained from the CMB itself; both the CMB dipole and degree-scale anisotropies provide a well-calibrated signal with precisely the desired spectrum. Scan-synchronous noise and systematic effects associated with the solid angle of the beam sidelobes limit the accuracy of the dipole calibration to $\sim 15 \%$. As described in Masi et al. (2006), cross-correlation with the WMAP data (Bennett et al. 2003b) provides an absolute calibration uncertainty of $1.8 \%$ on degree angular scales, including the uncertainty in the WMAP calibration. The precision of the B03 calibration is limited by the relatively low signal-to-noise ratio of the WMAP maps. The relative calibrations of the B03 detectors are obtained from cross-correlations of single-channel temperature maps and are accurate to $0.4 \%$.

The error in the in-flight pointing reconstruction is estimated from the apparent angular size of the extragalactic point sources in the B03 CMB fields. The effective beams are consistent with the physical optics model of the optical system, convolved with a $2.4 \mathrm{rms}$ Gaussian pointing jitter. These data result in a 0.23 FWHM statistical uncertainty in the amplitude of the jitter.

\section{ANALYSIS}

As a probe of the robustness of both the data and its analysis, the BOOMERANG team has implemented two fully independent analyses of the B03 TOD. The two pipelines, one centered in North America (NA) and one in Italy (IT), are described in detail in Masi et al. (2006), C. R. Contaldi et al. (2006, in preparation), Jones et al. (2006), de Gasperis et al. (2005), and references therein. In this section we summarize the general approach to signal, noise, and angular power spectrum estimation.

\subsection{Signal/Noise Estimation}

Estimates of the signal on the sky and noise in the time streams are obtained using an iterative procedure similar to that applied to the B98 temperature data (Prunet et al. 2001; Netterfield et al. 2002; Ruhl et al. 2003). Least-squares maps of the Stokes $I, Q$, and $U$ parameters are generated from a combined analysis of the data from all eight $145 \mathrm{GHz}$ PSBs, corresponding to roughly $3 \times 10^{8}$ time domain samples. The data from each channel are weighted by their sensitivity and combined during pixelization to decorrelate the linear Stokes parameters at each pixel. The Stokes parameter maps are generated at $\simeq 3.4$ resolution, resulting in approximately 377,000 spatial pixels.

The NA team divides the time stream into 200 noise-stationary subsets. The noise power spectra are calculated from each of these chunks using the converged estimate of the signal derived from the full set of data. These noise spectra are in general biased due to the finite signal-to-noise ratio in the signal estimate. Each of the noise spectra are corrected for this bias using an ensemble of signal and noise Monte Carlos. The IT analysis assumes stationarity of the noise over the course of the flight. While the NA approach is more general, the IT analysis benefits from a reduction in the sample variance of the noise estimate in the time domain. Each team employs a self-consistent treatment of the correlated noise between channels. Details regarding the two signal and noise estimation pipelines are provided in Jones et al. (2006) and de Gasperis et al. (2005). 


\subsection{Power Spectrum Estimation}

Both the NA and IT analysis pipelines employ variations of the pseudo- $C_{l}$ Monte Carlo approach to power spectrum estimation introduced in Hivon et al. (2002). Each incorporates a polarized implementation of a general least-squares signal estimator. The IT team uses a traditional pseudo- $C_{l}$ approach using either $1 / N_{\mathrm{obs}}^{1 / 2}$ or uniformly weighted masks to derive the temperature power spectrum, while a cross-correlation technique similar to that described in Tristram et al. (2005a) and Polenta et al. (2005) is used for the polarization analysis. Unlike these approaches, the NA estimator of the temperature and polarization power spectra is based on a hybrid auto- and cross-correlation technique adapted for multiple data sets characterized by partially overlapping sky coverage (C. R. Contaldi et al. 2006, in preparation).

As described in Hivon et al. (2002), the Monte Carlo approach approximates the spherical harmonic transform of the heuristically weighted data, $\tilde{C}_{l}^{\mathrm{TT}}$, as a convolution over the underlying power spectrum, $C_{l^{\prime}}^{\mathrm{TT}}$, with an additive noise term, $\tilde{N}_{l}$,

$$
\tilde{C}_{l}^{\mathrm{TT}}=\sum_{l^{\prime}} K_{l l^{\prime}} B_{l^{\prime}}^{2} F_{l^{\prime}} C_{l^{\prime}}^{\mathrm{TT}}+\tilde{N}_{l} .
$$

The coupling kernel, $K_{l l^{\prime}}$, is derived analytically from the transform of the weighted mask that has been applied to the data. The window function, $B_{l}$, is the transform of the effective beam on the sky and approximates the convolution over the Stokes parameters appearing in equation (1). The transfer function, $F_{l}$, is a symmetrized approximation of the combined effect of the scan strategy and time-domain processing on the sensitivity to a given angular scale.

The transfer function is determined from an ensemble of signalonly Monte Carlo simulations, while the noise pseudopower spectrum, $\tilde{N}_{l}$, is obtained from an ensemble of noise-only simulations. Both the signal and noise simulations treat the flagged portions of the time stream in exactly the same manner as the actual data.

\subsection{The B03 Temperature Power Spectrum}

The Monte Carlo approach to power spectrum estimation represents an approximate treatment of the noise covariance matrix of the map and therefore generally results in larger statistical uncertainties than that achieved by direct methods (Borrill 1999). The sensitivity achieved by a Monte Carlo estimate of the power spectrum is determined by the properties of the noise and the weighting that are applied to the data.

When analyzing a single map generated from the full set of B03 data, a complication arises in the selection of the weighting that is applied to the mask. In the limit of white (that is, pixeluncorrelated) noise, the optimal power spectrum estimate of a noise-dominated signal is obtained by weighting the pixels according to $1 / N_{\text {obs }}^{1 / 2}$. For the combined set of shallow and deep data, this approach is virtually equivalent to analyzing only the deep region. Conversely, in the signal-dominated regime a uniform weighting will reduce (to the extent possible) the sample variance in the bandpower estimates. The choice of weighting reflects a trade-off between the optimality of error bars at low and high multipoles. For the B03 data the angular power spectrum is signal dominated at multipoles below $l \lesssim 800$, as indicated in column (4) of Table 2 .

The IT temperature power spectrum is derived from an analysis of the combined shallow and deep field data, using either uniform or $1 / N_{\mathrm{obs}}^{1 / 2}$ pixel weighting, depending on the spatial scales of interest. The agreement between the NA and IT analyses, as well as with the B98 results reported in Ruhl et al. (2003), is illustrated in Figure 2. These results, representing two independent data sets and three independent analysis pipelines, are indicative of the robustness of the result.
The NA pipeline addresses the problem of heuristic weighting of data with nonuniform coverage by treating the shallow and deep TOD separately. These data subsets, which have roughly uniform noise per pixel, exhibit partially correlated signal and statistically independent noise. A uniform weighting is applied to the shallow data, while the deep data are noise weighted.

Estimates of the underlying power spectra are derived from the joint analysis of the two autospectra and the cross-power spectrum of the shallow and deep data, using a diagonal approximation to the quadratic Fisher matrix estimator. The inclusion of both the auto- and cross-power spectra allows nearly optimal errors to be obtained over the full range of angular scales, while computing the complete Fisher matrix in a self-consistent fashion for all the band powers (temperature and polarization) simultaneously (C. R. Contaldi et al. 2006, in preparation).

The full resolution B03 spectrum is displayed with the best-fit concordance $\Lambda$ CDM model in the top panel of Figure 3 (MacTavish et al. 2006). The maximum likelihood band powers, the diagonal components of the correlation matrix, and the ratio of sample to noise variance are presented in Table 2. Neighboring bins are anticorrelated at the $\simeq 12 \%-20 \%$ level.

The B03 data improve on the published measurements of the temperature angular power spectrum primarily over the third peak, at angular scales corresponding to the photon mean free path at the surface of last scattering. All of the B03 temperature and polarization power spectra, inverse Fisher matrices, window functions, and explanatory supplements are publicly available on the BOOMERANG web servers. ${ }^{22}$

\subsection{Internal Consistency Tests}

As a check on the internal consistency of the data, we perform two jackknife tests in which the TOD are divided into two halves, with Stokes $I, Q$, and $U$ maps generated from each half independently. Temperature and polarization power spectra are then computed from the difference of the resulting maps.

The channel jackknife measures the difference between maps generated from the two halves of the focal plane. Each side of the focal plane accommodates two pairs of PSBs, allowing complete characterization of the three linear Stokes parameters. The sky coverage, filtering, and data flagging of this jackknife test are nearly identical to that of the full data set.

The temporal jackknife separates the data into a first and second half. The shallow and deep region scans are distributed between the data subsets such that each include roughly equal sky coverage. As discussed in Masi et al. (2006) and Jones (2005), B03 experienced a dramatic $(\simeq 9 \mathrm{~km})$ loss of altitude over the course of the flight. The first-half/second-half test was chosen to provide a check that is maximally sensitive to systematic effects related to the altitude drop, such as responsivity drifts, a degradation in the accuracy of the pointing reconstruction, or atmospheric contamination.

The noise properties and therefore the inverse noise filtering of the signal vary slightly from channel to channel. When the data are processed in the time domain, this asymmetry, combined with the channel-specific flagging of the TOD, results in a nonzero difference spectrum even in a noiseless observation free of systematic effects. The temporal jackknife is similarly affected; the observations are not symmetric for the two subsets of data, resulting in subtle differences in the signal processing. For both the channel-based and temporal divisions of the data, this offset is easily characterized in the Monte Carlo analysis by performing

\footnotetext{
${ }^{22}$ See http://cmb.phys.cwru.edu/boomerang/index.html and http://oberon roma1.infn.it/boomerang/b2k.
} 
TABLE 2

BoOmerang 2003 Temperature Power Spectrum

\begin{tabular}{|c|c|c|c|c|c|c|c|c|}
\hline $\begin{array}{c}l_{b} \\
(1)\end{array}$ & $\begin{array}{c}\mathcal{C}_{b} \\
\left(\mu \mathrm{K}^{2}\right) \\
(2)\end{array}$ & $\begin{array}{c}\Delta \mathcal{C}_{b} \\
\left(\mu \mathrm{K}^{2}\right) \\
(3)\end{array}$ & $\begin{array}{c}\mathcal{C}_{b} / \mathcal{N}_{b} \\
\quad(4)\end{array}$ & $\begin{array}{c}(\mathrm{WX}-\mathrm{YZ}) / 2 \\
\left(\mu \mathrm{K}^{2}\right) \\
(5)\end{array}$ & $\begin{array}{c}(1 \mathrm{st}-2 \mathrm{nd}) / 2 \\
\left(\mu \mathrm{K}^{2}\right) \\
(6)\end{array}$ & $\begin{array}{c}\Delta \mathcal{C}_{b}^{\text {sys }} \\
\left(\mu \mathrm{K}^{2}\right) \\
(7)\end{array}$ & $\begin{array}{c}\Delta \mathcal{C}_{b}^{\text {tot }} \\
\left(\mu \mathrm{K}^{2}\right) \\
(8)\end{array}$ & $\begin{array}{c}\Delta B_{l} / B_{l} \\
\quad(9)\end{array}$ \\
\hline 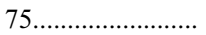 & 2406 & 410 & 12.28 & $10 \pm 11$ & $6 \pm 11$ & 4.1 & 410 & 0.051 \\
\hline $125 \ldots \ldots \ldots \ldots \ldots \ldots$ & 4167 & 464 & 28.25 & $1 \pm 6$ & $-11 \pm 6$ & 6.8 & 464 & 0.036 \\
\hline $175 \ldots \ldots \ldots \ldots \ldots$ & 5250 & 459 & 117.66 & $8 \pm 6$ & $-12 \pm 6$ & 9.5 & 459 & 0.019 \\
\hline $225 \ldots \ldots \ldots \ldots \ldots$ & 5306 & 420 & 132.13 & $-10 \pm 5$ & $-8 \pm 8$ & 12.2 & 420 & 0.005 \\
\hline $275 \ldots \ldots \ldots \ldots$ & 5235 & 372 & 125.39 & $-4 \pm 5$ & $-4 \pm 8$ & 14.9 & 372 & 0.005 \\
\hline $325 \ldots \ldots \ldots \ldots$ & 3323 & 236 & 72.60 & $8 \pm 7$ & $0 \pm 8$ & 17.6 & 236 & 0.009 \\
\hline $375 \ldots \ldots \ldots \ldots \ldots \ldots$ & 1884 & 144 & 12.89 & $0 \pm 7$ & $-9 \pm 7$ & 20.3 & 145 & 0.008 \\
\hline $425 \ldots \ldots \ldots \ldots \ldots \ldots$ & 1870 & 136 & 7.71 & $7 \pm 8$ & $39 \pm 13$ & 23.0 & 137 & 0.008 \\
\hline $475 \ldots \ldots$ & 2172 & 149 & 5.97 & $6 \pm 10$ & $42 \pm 15$ & 25.7 & 151 & 0.009 \\
\hline $525 \ldots \ldots \ldots \ldots \ldots$ & 2338 & 159 & 4.45 & $12 \pm 12$ & $3 \pm 14$ & 28.4 & 161 & 0.010 \\
\hline $575 \ldots \ldots \ldots \ldots \ldots$ & 2429 & 165 & 3.37 & $-13 \pm 11$ & $22 \pm 17$ & 31.1 & 167 & 0.010 \\
\hline $625 \ldots \ldots \ldots \ldots \ldots \ldots$ & 1806 & 144 & 1.97 & $5 \pm 16$ & $54 \pm 22$ & 33.8 & 147 & 0.011 \\
\hline 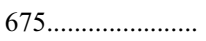 & 1663 & 146 & 1.45 & $23 \pm 21$ & $-4 \pm 20$ & 36.5 & 150 & 0.011 \\
\hline $725 \ldots \ldots \ldots \ldots \ldots$ & 2117 & 177 & 1.41 & $17 \pm 25$ & $47 \pm 30$ & 39.2 & 181 & 0.011 \\
\hline $775 \ldots \ldots$ & 2440 & 206 & 1.28 & $-9 \pm 28$ & $56 \pm 38$ & 41.9 & 210 & 0.012 \\
\hline $825 \ldots \ldots \ldots \ldots \ldots \ldots$ & 1968 & 207 & 0.90 & $-33 \pm 31$ & $154 \pm 53$ & 44.6 & 211 & 0.014 \\
\hline 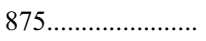 & 1915 & 221 & 0.78 & $6 \pm 43$ & $73 \pm 56$ & 47.3 & 226 & 0.016 \\
\hline $925 \ldots \ldots \ldots \ldots \ldots \ldots$ & 1545 & 223 & 0.61 & $-11 \pm 56$ & $104 \pm 67$ & 50.0 & 228 & 0.018 \\
\hline 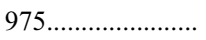 & 842 & 209 & 0.37 & $-83 \pm 66$ & $-20 \pm 71$ & 52.7 & 215 & 0.021 \\
\hline $1025 \ldots \ldots \ldots \ldots \ldots$ & 1034 & 247 & 0.38 & $-28 \pm 93$ & $90 \pm 97$ & 55.4 & 253 & 0.023 \\
\hline 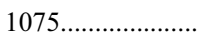 & 989 & 289 & 0.32 & $11 \pm 129$ & $0 \pm 122$ & 58.1 & 294 & 0.026 \\
\hline $1125 \ldots \ldots \ldots \ldots \ldots$ & 1108 & 343 & 0.32 & $-81 \pm 159$ & $-31 \pm 151$ & 60.8 & 348 & 0.028 \\
\hline $1225 \ldots \ldots \ldots \ldots \ldots$ & 691 & 224 & 0.13 & $-89 \pm 130$ & $224 \pm 128$ & 66.2 & 233 & 0.034 \\
\hline $1400 \ldots \ldots \ldots \ldots \ldots$ & 1157 & 449 & 0.06 & $-610 \pm 342$ & $702 \pm 301$ & 75.7 & 455 & 0.045 \\
\hline
\end{tabular}

Notes.-The angular power spectrum of the B03 temperature data and the results of the consistency tests described in $\S 3.4$. The band powers, $\mathcal{C}_{b}=C_{b} l_{b}\left(l_{b}+1\right) / 2 \pi$, and their errors are in units of square microkelvins. The full set of temperature and polarization angular power spectra and the associated covariance matrices and window functions are publicly available on the BOOMERANG web servers, http://cmb.phys.cwru.edu/boomerang/index.html and http://oberon.roma1.infn.it/boomerang/b2k. Neighboring bins are anticorrelated at the $12 \%-20 \%$ level, depending on the multipole bin. The errors, $\Delta \mathcal{C}_{b}$, are taken from the diagonal elements of the covariance matrix. The diagonal elements of the covariance matrix can be approximated by the formula $\Delta C_{b} \simeq\left\{2 /\left[\left(2 l_{b}+1\right) \Delta l f_{\text {sky }} \mathcal{F}_{b}\right]\right\}\left(C_{b}+N_{b}\right)$, where $\mathcal{F}_{b} \leq 1$ is a parameterization of the mode loss resulting from the filtering applied to the data. The $N_{b}$ term represents the (binned and deconvolved) power spectrum of the noise, as determined from noise only simulations. Therefore, the ratio $C_{b} / N_{b}$ is indicative of the relative contribution of sample and noise variance for a given band power. The band powers obtained from the two consistency tests described in $\S$ 3.4 are shown in col. (5) and (6). We have subtracted the ensemble-averaged power spectra derived from the jackknifes of the signal and noise Monte Carlos. The $\chi^{2}$ to zero for the channel (temporal) jackknife is $\chi^{2}=20.8(55.5)$ for 24 degrees of freedom. As discussed in $\S 3.4, \Delta \mathcal{C}_{b}^{\text {sys }}$ is an upper limit on the systematic contribution to the uncertainty in each band, derived from the jackknife tests. The adjacent column, $\Delta \mathcal{C}_{b}^{\text {tot }}$, is the quadrature sum of the systematic and statistical contributions to the error on the band power estimates. Finally, $\Delta B_{l} / B_{l}$ represents the $1 \sigma$ uncertainty on the beam window function, which is plotted as the solid line enveloping the largest effects in Fig. 5.

the same consistency test on an ensemble of simulations. The ensemble average of these simulated difference spectra are directly subtracted from those derived from the data.

The high signal-to-noise ratio with which the temperature anisotropies are detected is evident in the spectra of the differenced data, which are shown in the bottom two panels of Figure 3. The first of these is the spectrum of the difference generated from the channel-based division; the second is the spectrum derived from the temporal division. While the former (with a $\chi^{2}=20.8$ for 24 degrees of freedom) passes the jackknife test, the latter clearly fails $\left(\chi^{2}=55.5\right)$.

The failure of this jackknife test suggests that the temperature data are contaminated at the level of $2-10 \mu \mathrm{K}$. Guided by these results, we assume that the source of the contamination can be characterized by a semistationary signal having a spatial power spectrum that falls more gradually than $l^{-2}$ (as would be consistent with a Kolmogorov spectrum of atmospheric fluctuations). The amplitude of this component is taken to be that, which when added in quadrature to the diagonal elements of the noise covariance matrix, drives the reduced $\chi^{2} / \nu \rightarrow 1$ for the first-half/ second-half jackknife. As shown in Table 2, this effect contributes at most $\sim 5 \%$ to the uncertainty in any given bin. The CMB results are therefore only weakly dependent on the assumed shape of the spectrum of the systematic contribution.

\subsection{Propagation of Systematic Errors}

Uncertainties in the calibration and low-level analysis of the data propagate in a complex way from the TOD to the band-power estimates. For the $\mathrm{B} 03$ analysis the principle uncertainties are the determination of the in-flight transfer functions, the relative calibrations, the polarization efficiencies, and the effective beam size.

A very powerful feature of the Monte Carlo approach is the ease with which such effects can be modeled as they appear in the time domain, and then propagated through to the power spectra. In particular, we investigate the impact of the uncertainty in the polarization leakage $(\epsilon, \pm 3 \%)$, the PSB orientation in the focal plane $\left(\psi, \pm 2^{\circ}\right)$, and the relative calibration $(s, \pm 0.4 \%)$ that appear in equations (1) and (2).

In addition, we probe the impact of uncertainty on the in-flight system transfer function used to deconvolve each of the bolometer time streams at the earliest stage of the analysis. The system transfer function is the product of the bolometer response and that of the readout electronics. The former is well described by a single-pole time constant $(\tau, \pm 10 \%)$, with the uncertainty a 

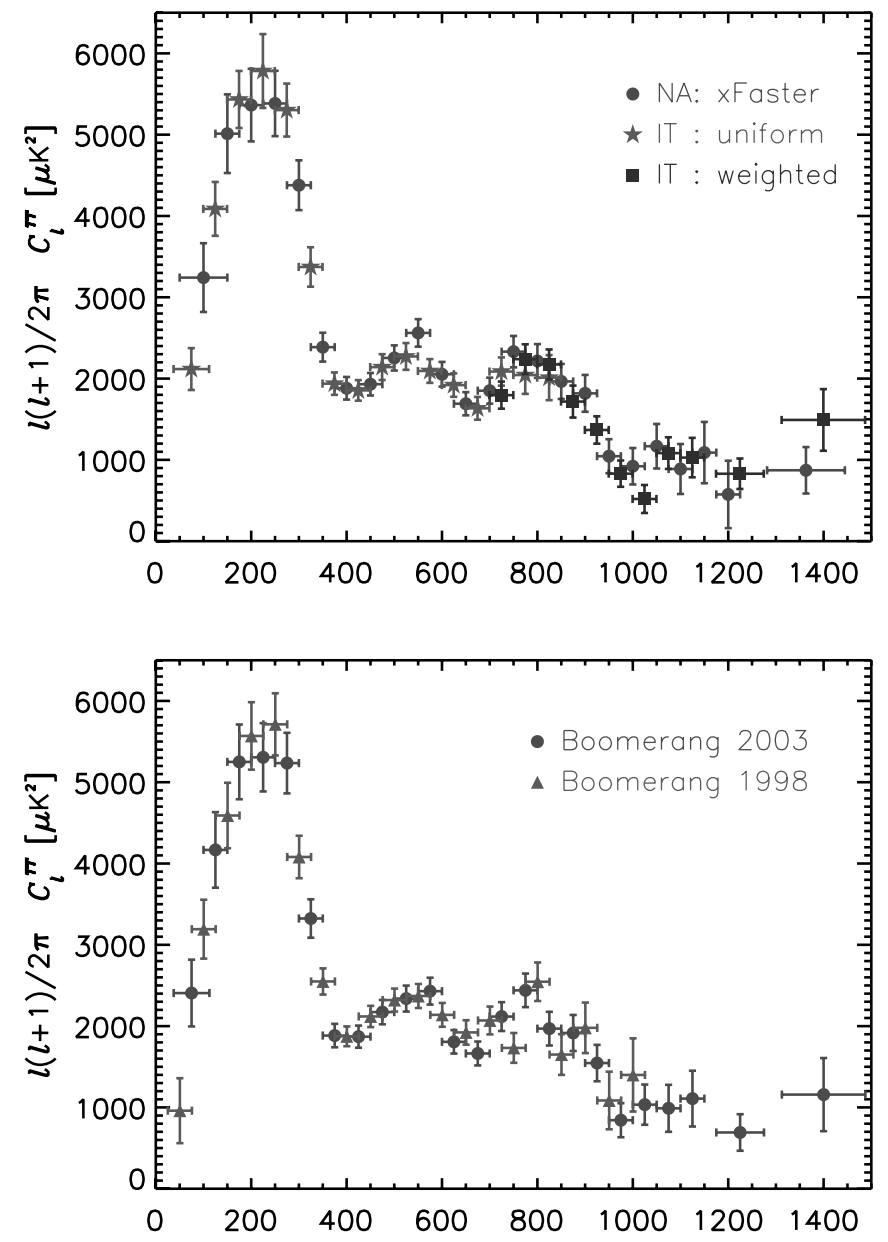

Fig. 2.-Top: Comparison of the temperature angular power spectrum derived from the B03 data using the NA pipeline (xFaster, circles) and the IT pipeline, the latter using both uniform (stars) and noise-weighted (squares) masks. For the IT results uniform weighting of the combined shallow and deep data results in nearly optimal error bars in the signal-dominated regime, while the $1 / N_{\text {obs }}^{1 / 2}$ pixel weighting is required to achieve this sensitivity in the noise-dominated regime. The NA spectrum is derived using a hybrid auto- and cross- correlation technique that achieves nearly optimal errors across the full multipole range. Bottom: Comparison of the angular power spectrum derived from the B98 data (Ruhl et al. 2003) and the B03 results reported here. The 2003 coverage is a subset of the region observed in 1998, so the signal is completely correlated between the two data sets. The binning of the B03 data in the bottom panel is shifted by half of a bin relative to the top panel. [See the electronic edition of the Journal for a color version of this figure.]

result of the limited accuracy of our knowledge of the in-flight loading conditions (see Jones [2005] and Masi et al. [2006] for further discussion of this topic). The transfer function of the electronics is observed to be highly stable over the range of temperatures experienced during the flight and is measured to much higher precision.

A simulated set of TOD is generated for each B03 channel using a realization of the best-fit concordance $\Lambda$ CDM cosmology while randomly varying each of these instrumental parameters about their nominal values. An ensemble of these TODs are processed through the NA analysis pipeline, and the distribution of the resulting band powers are used to propagate uncertainties on the instrumental parameters to systematic error bars on the band power estimates. The result of this analysis, shown in Figure 4 , indicates the amplitude of the systematics that result from uncertainties in the characterization of the receiver.

As discussed in detail in Page et al. (2003a), limited knowledge of the beam results in uncertainty in the experimental window

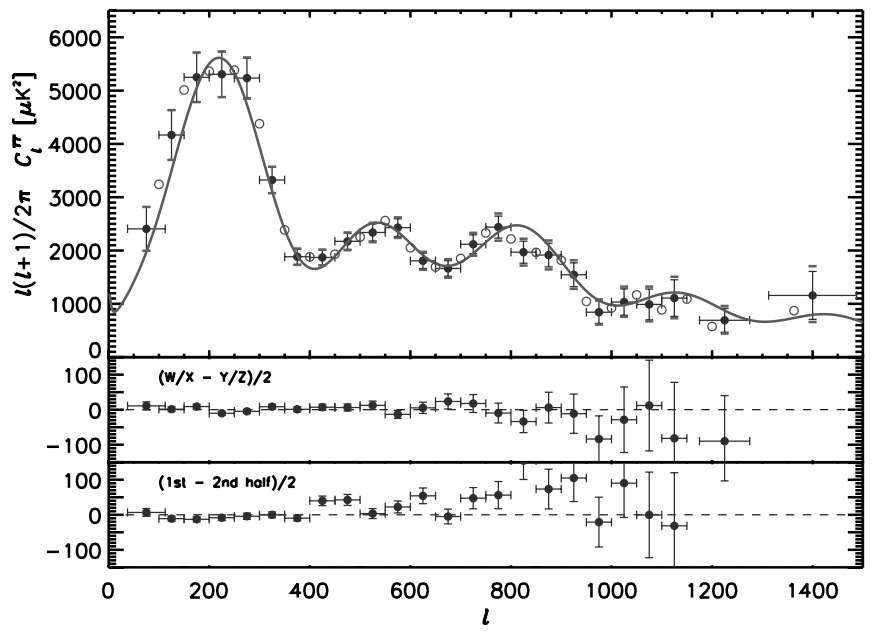

FIG. 3.- Temperature angular power spectrum, $\mathcal{C}_{l}^{\mathrm{TT}}$, derived from the B03 data. The open points represent an alternate binning and should not be interpreted as having statistical weight beyond that of the nominally binned data, which are shown with error bars. The anticorrelations between neighboring bands range from the $12 \%$ to $20 \%$ level. The envelope of the beam uncertainty is indicated by ticks bracketing the $1 \sigma$ error bars for each of the band power estimates. As discussed in $\S 3.5$, these limits indicate the amount of "tilt" to the spectrum that is allowed by the beam uncertainty and should not be interpreted as an additional uncorrelated error in each bin. The solid line is the concordance $\Lambda \mathrm{CDM}$ model that best fits all published CMB data, including the B03 temperature and polarization results. The power spectra of the consistency checks described in the text are shown in the bottom two panels. [See the electronic edition of the Journal for a color version of this figure.]

function. For B03 this window function is effectively normalized at degree angular scales through cross-correlation with WMAP, and is necessary to link the calibration to both higher and lower multipoles. Misestimation of this window function generally results in a (highly correlated) distortion of the power spectrum. The uncertainty in the B03 beam results from two distinct effects,

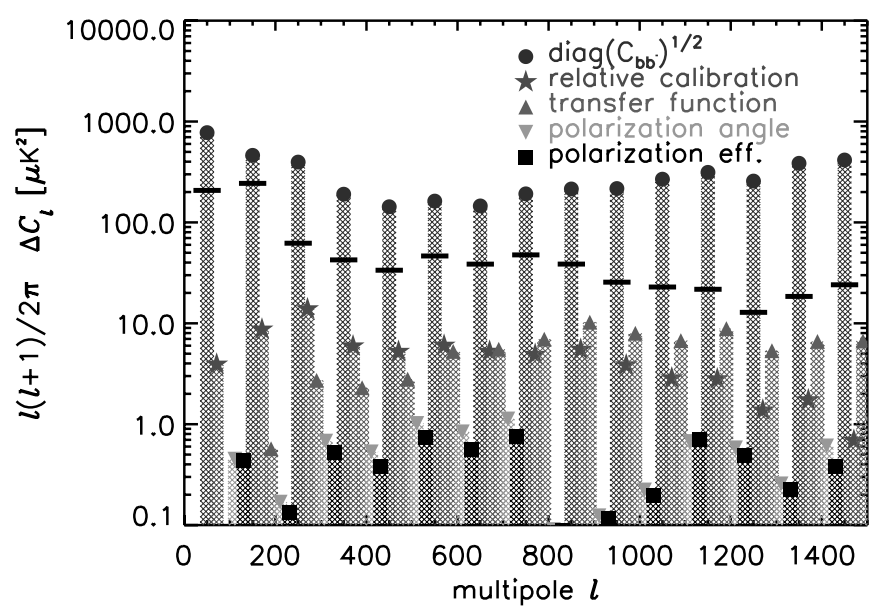

FIG. 4.-Propagation of instrumental calibration errors to the band power estimates. The amplitude of the statistical uncertainties (sample variance plus instrumental noise) for each bin are shown with circles. The contributions from the relative calibration, $s \pm 0.4 \%$, the in-flight transfer function, $\tau \pm 10 \%$, the polarization angle, $\psi \pm 2^{\circ}$, and the polarization efficiency, $\epsilon \pm 3 \%$, are shown for reference. The horizontal ticks indicate the effect of uncertainty on the beam. Note that these effects are highly correlated between bins and therefore are not properly treated by quadrature addition to the statistical uncertainty in a given bin. Since the correlation structure of these errors is known, they are more properly treated as nuisance parameters and, as such, are marginalized over during the cosmological parameter estimation (Bridle et al. 2002). For the B03 parameter extraction, only the beam error and calibration uncertainty are treated in this manner (MacTavish et al. 2006). [See the electronic edition of the Journal for a color version of this figure.] 


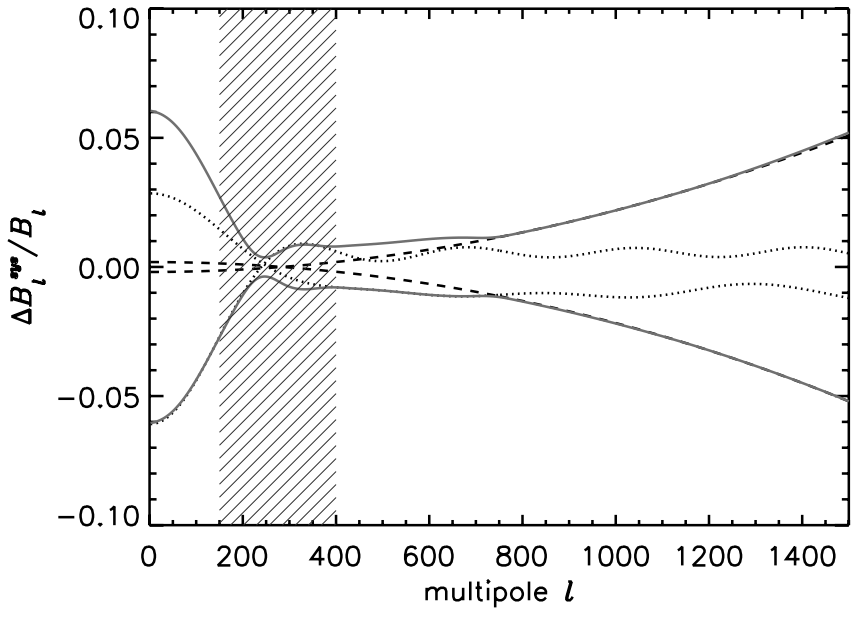

FIG. 5.-Effect of beam uncertainty on the B03 window function. The knowledge of the beam on the sky is limited by two effects: uncertainty in the amplitude of the error in the pointing reconstruction and limited knowledge of the near sidelobe structure. These effects contribute to an uncertainty in the experimental window function that extends the calibration over a wide range of angular scales (Page et al. 2003a). The hatched region shows the angular scales over which the cross-calibrations with $W M A P$ and B98 are applied, effectively normalizing the window functions over that range. Uncertainty in the pointing jitter, the effects of which are shown as dashed lines, dominate at small spatial scales. Uncertainty in the near sidelobe structure, or equivalently the contribution of the sidelobes to the beam solid angle, dominates the effect on the largest scales. The solid orange line is the envelope defined by the largest of these uncertainties. [See the electronic edition of the Journal for a color version of this figure.]

(1) error in the pointing reconstruction and (2) uncertainty in the contribution of the near sidelobe structure to the beam solid angle.

The error in BOOMERANG's pointing reconstruction is well described by an isotropic Gaussian jitter. The effective beam on the sky is the convolution of the physical beam with this jitter, the amplitude of which is derived from fits to the five brightest extragalactic sources in the CMB field. As described in $\S 2.3$, these observations provide a measurement of the jitter amplitude with an uncertainty of 0.23 . Misestimation of the jitter amplitude will result in a bias that is equal to the ratio of the assumed and underlying window functions,

$$
\frac{W_{l}^{\prime}}{W_{l}}=\frac{W_{l}^{0} e^{-\left(\sigma_{j}+\Delta \sigma_{j}\right)^{2} l(l+1)}}{W_{l}^{0} e^{-\sigma_{j}^{2} l(l+1)}}=e^{-\sigma_{j}^{2}\left(\delta^{2} \pm 2|\delta|\right) l(l+1)},
$$

where $W_{l}^{0}$ is the window function of the physical beam, $\sigma_{j}$ is the rms pointing jitter, and $\delta=\Delta \sigma_{j} / \sigma_{j}$ is the fractional error on the jitter. The envelope of the $2 \sigma$ limits that correspond to $\delta \simeq \pm 10 \%$ are shown as ticks bracketing the error bars in Figure 3. The error on the window function, given both a fiducial beam model and the calibration, are shown in the dashed lines in Figure 5.

As discussed in Masi et al. (2006), the beam model of the sidelobes is verified experimentally at the $-25 \mathrm{~dB}$ level. In order to quantify the effect of the sidelobe structure below this level on the window function, we calculate the variation in the window function that results from perturbing the modeled beam within the limits of the preflight beam measurements. Uncertainty in the contribution of the near sidelobe structure to the beam solid angle has the largest effect on the B03 window function at the lowest multipoles, due to the method of calibration. This effect is shown as the dotted line in Figure 5.

The effects of both instrumental calibration and beam uncertainty are highly correlated from bin to bin and have a known spectrum, or are only weakly dependent on the underlying spectrum of the signal. As such, they are both properly treated as nuisance parameters rather than independent contributions to the uncertainty in each bin. In the B03 parameter estimation, the calibration and beam uncertainty are treated in this manner, following closely the prescription of Bridle et al. (2002).

\subsection{Foreground Contamination}

Galactic microwave emission can potentially contaminate observations of the CMB. At $150 \mathrm{GHz}$, the thermal emission of interstellar dust grains is expected to be the dominant component of Galactic emission (Brandt et al. 1994; de Oliveira-Costa 2005; Masi et al. 2001). Therefore, any Galactic contamination in the $145 \mathrm{GHz}$ B03 data is expected to exhibit some spatial correlation with existing infrared surveys. The spectrum of the Galactic continuum emission is distinct from that of the CMB, allowing multifrequency observations to discriminate between the cosmological signal and local foregrounds. For this reason B03 made simultaneous observations at 245 and $345 \mathrm{GHz}$.

Using the method described in de Oliveira-Costa et al. (1999), we quantify the level of foreground contamination in the B03 $145 \mathrm{GHz}$ data by cross-correlating the Stokes $I$ parameter map with the Schlegel-Finkbeiner-Davis (SFD) dust map. The SFD map is a composite of the all-sky Diffuse Infrared Background Experiment (DIRBE) and Infrared Astronomical Satellite (IRAS) $100 \mu \mathrm{m}$ surveys (Hauser et al. 1998; Neugebauer et al. 1984; Finkbeiner et al. 1999; Schlegel et al. 1998).

We estimate the uncertainty on the correlation by applying the same analysis to $50 \mathrm{~B} 03$ noise realizations. Before calculating the correlations, we resample the DIRBE/IRAS data with the B03 scan pattern and filtering and convolve both the DIRBE/IRAS data and the noise with a $1^{\circ}$ Gaussian. As a consequence our results are insensitive to the zero point of the $100 \mu \mathrm{m}$ maps. Using this procedure, we find no statistically significant correlation between the $145 \mathrm{GHz}$ B03 and $100 \mu \mathrm{m}$ data in either the B03 deep $(b \lesssim-30)$ or shallow $(b \lesssim-20)$ fields from which the $\mathrm{CMB}$ power spectrum is derived.

The same analysis applied to the 245 and $345 \mathrm{GHz}$ B03 data results in very clear correlations with the $100 \mu \mathrm{m}$ SFD map. The amplitude of these correlations, described in more detail in Masi et al. (2006), provides an empirical scaling of the observed surface brightness of the $100 \mu \mathrm{m}$ dust to $145 \mathrm{GHz}$. When applied to the SFD map, this empirical scaling implies rms fluctuations due to unpolarized dust of about $1 \mu \mathrm{K}$ in the B03 deep region at the angular scales of interest. This is roughly 2 orders of magnitude below the $\mathrm{CMB}$ contribution and will result in a correspondingly negligible impact on the estimate of the CMB power spectrum.

\subsection{Features in the Temperature Power Spectrum}

A series of acoustic peaks is readily apparent in the B03 power spectrum. As has become common practice (see, e.g., de Bernardis et al. 2002; Ruhl et al. 2003; Page et al. 2003b), we calculate a model-independent characterization of the location, amplitude, and significance of the features in the power spectrum through a comparison of the goodness of fit of a parabola (or Gaussian) to each set of five contiguous bins. ${ }^{23}$ In this work, we fit a Gaussian to the band powers in the vicinity of the first peak, and a parabola to the other features.

As is well known, the likelihoods of the band powers are not Gaussian distributed (Bond et al. (1998, 2000). We therefore transform to the "offset lognormal" variables whose likelihood

23 While the result is relatively insensitive to the number of bins that are fit, as the subsets get larger than the characteristic size of the features in the spectrum, the reduced $\chi^{2}$ clearly will degrade. Five bins is found to be the largest set that does not result in a poor goodness-of-fit statistic over the full range in $l$. 

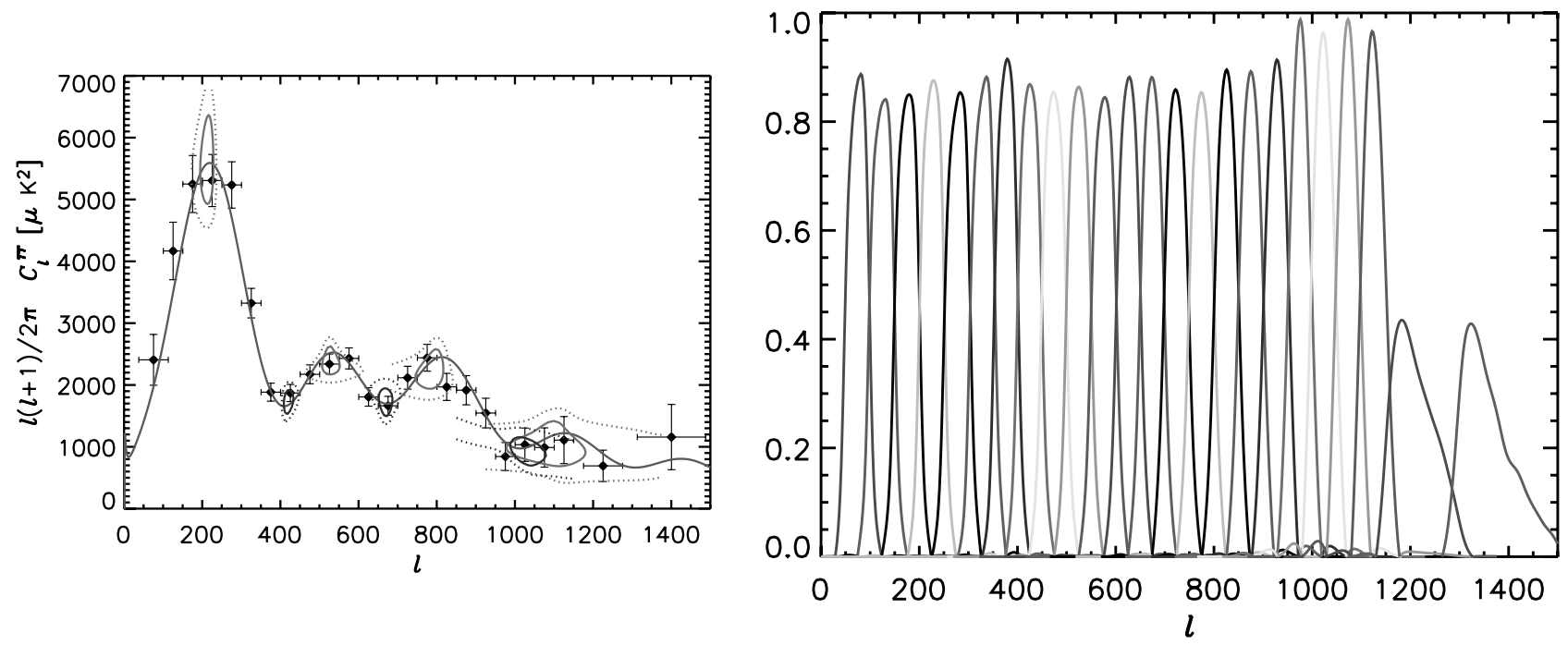

FIG. 6. - Left: CMB spectrum with the 1 and $2 \sigma \Delta \chi^{2}$ contours for the fits shown for the features determined to have positive or negative curvature. The likelihoods have been marginalized over the curvature parameter. The fourth "peak" only marginally favors negative curvature over a flat band power and is not considered a detection. Right: Window functions used in the generation of the band power estimates. [See the electronic edition of the Journal for a color version of this figure.]

distributions are better approximated by a Gaussian (Bartlett et al. 2000; Bond et al. 2000). The transformation is a simple one,

$$
Z_{b} \equiv \ln \left(\mathcal{C}_{b}+x_{b}\right)
$$

where the noise offsets, $x_{b}$, are determined from the (binned and deconvolved) $\tilde{N}_{l}$ as derived from the noise Monte Carlos. The inverse Fisher (covariance) matrix must similarly be transformed,

$$
\left(F^{Z}\right)_{b b^{\prime}}^{-1}=\frac{F_{b b^{\prime}}^{-1}}{\left(\mathcal{C}_{b}+x_{b}\right)\left(\mathcal{C}_{b^{\prime}}+x_{b^{\prime}}\right)} .
$$

In addition, the models to be fit are binned into band powers according to the same instrumental window functions, $W_{l}$ (shown in Fig. 6) that are applied to the raw spectra,

$$
\mathcal{C}_{b}^{X X} \equiv \frac{\mathcal{I}\left(W_{l}^{b} \mathcal{C}_{l}^{X X}\right)}{\mathcal{I}\left(W_{l}^{b}\right)},
$$

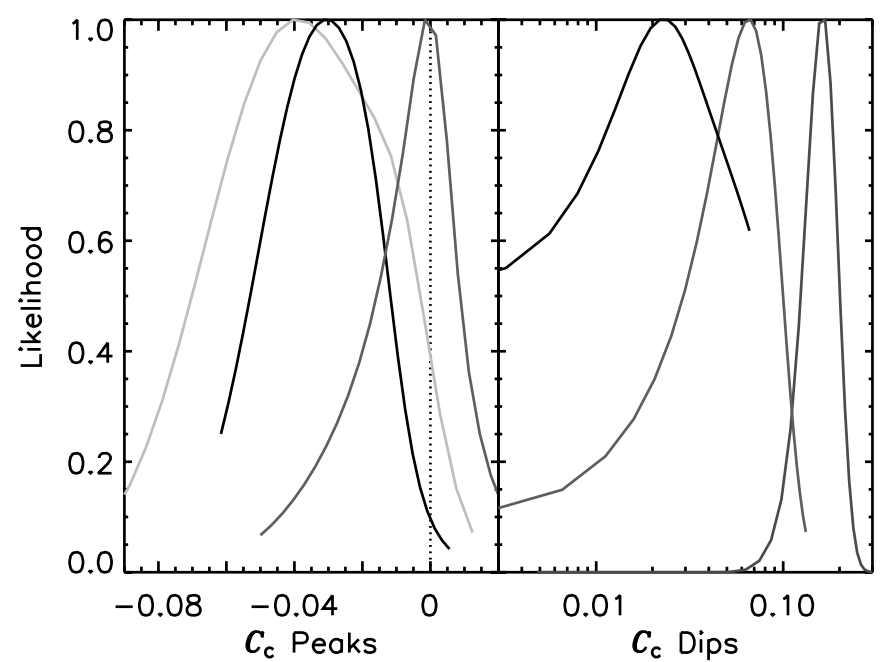

where the logarithmic binning operator is defined as

$$
\mathcal{I}\left(f_{l}\right) \equiv \sum_{l} \frac{(l+1 / 2)}{l(l+1)} f_{l} .
$$

The parabolic model,

$$
\mathcal{C}_{l}^{m}=\mathcal{C}_{c}\left(l-l_{0}\right)^{2}+\mathcal{C}_{0}
$$

is similarly transformed using the offset lognormal approximation, and the three-dimensional likelihoods are calculated directly on a grid about each of the best-fit locations. The $\Delta \chi^{2}$ contours for the curvature-marginalized likelihoods are shown in Figure 6.

In previously published B98 data, the significance of a detection has been determined by the curvature of the likelihood at the peak of the distribution (de Bernardis et al. 2002; Ruhl et al. 2003). However, as is evident in Figure 7, the distributions are highly nonGaussian. We therefore determine the significance of the detections from the amplitude of the marginalized likelihood at zero curvature.

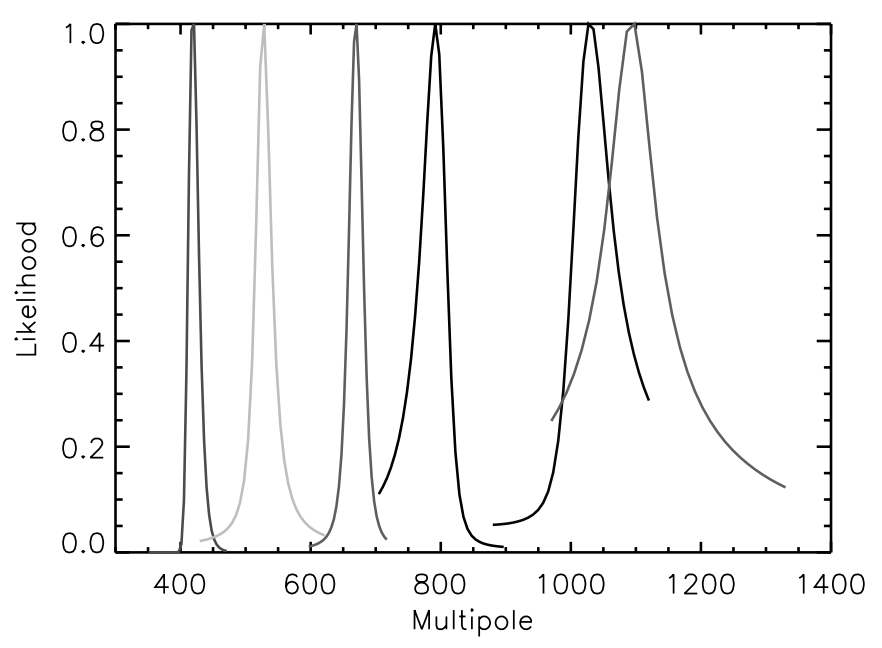

FIG. 7.-Marginalized likelihoods for the curvature parameter, $\mathcal{C}_{c},(l e f t)$ and the multipole, $l_{0}$, $($ right $)$ of each feature in the temperature power spectrum. All three dips in the

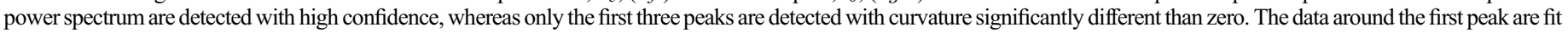

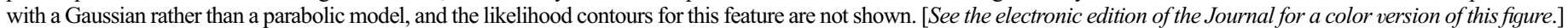


TABLE 3

Features in the Temperature Power Spectrum

\begin{tabular}{|c|c|c|c|c|c|c|}
\hline Feature & $l_{\mathrm{B} 03}$ & $\Delta T_{\mathrm{B} 03}^{2}$ & $l_{\mathrm{B} 98}$ & $\Delta T_{\mathrm{B} 98}^{2}$ & $l_{W M A P}$ & $\Delta T_{W M A P}^{2}$ \\
\hline 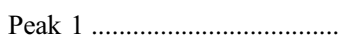 & $214_{-12}^{+9}$ & $5614_{-443}^{+450}$ & $217_{-10}^{+10}$ & $5551_{-443}^{+477}$ & $222_{-2}^{+3}$ & $5385_{-157}^{+147}$ \\
\hline 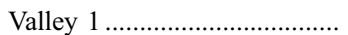 & $413_{-5}^{+10}$ & $1717_{-70}^{+133}$ & $411_{-7}^{+9}$ & $1870_{-120}^{+136}$ & $418_{-4}^{+5}$ & $1660_{-62}^{+62}$ \\
\hline Peak 2 & $529_{-6}^{+14}$ & $2419_{-128}^{+125}$ & $526_{-14}^{+17}$ & $2316_{-121}^{+119}$ & $530_{-8}^{+15}$ & $2404_{-64}^{+89}$ \\
\hline 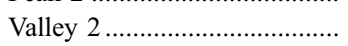 & $659_{-11}^{+12}$ & $1780_{-165}^{+131}$ & $(677)_{-29}^{+65}$ & $(1958)_{-170}^{+200}$ & $\ldots$ & $\ldots$ \\
\hline Peak 3 & $781_{-22}^{+11}$ & $2166_{-216}^{+165}$ & $(766)_{-43}^{+42}$ & $(2080)_{-227}^{+261}$ & $\ldots$ & $\cdots$ \\
\hline Valley 3 & $1015_{-23}^{+26}$ & $991_{-192}^{+137}$ & $\ldots$ & $\ldots$ & $\cdots$ & $\cdots$ \\
\hline 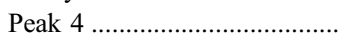 & $(1055)_{-56}^{+58}$ & $(1024)_{-271}^{+254}$ & $\ldots$ & $\ldots$ & $\ldots$ & $\ldots$ \\
\hline
\end{tabular}

Notes.-A comparison of the locations and amplitudes of the features in the temperature power spectrum derived from the B03, B98, and WMAP data sets. The values and $1 \sigma$ errors are obtained directly from the marginalized likelihood distributions. Values in parenthesis indicate a curvature parameter consistent with zero at the $2 \sigma$ level, or greater [that is, a marginalized likelihood for the curvature parameter for which $\left.\mathcal{L}\left(\mathcal{C}_{c}=0\right) \geq 2 \sigma\right]$. Note that the amplitude of the first peak in B03 and WMAP data are consistent by construction, given the method of calibration. This analysis has been performed on the binned WMAP data for better comparison with the BOOMERANG window functions. The full-resolution WMAP spectrum provides constraints on the first peak and dip locations that are stronger than (but also consistent with) those reported here. For the full resolution analysis of the first-year WMAP data, see Page et al. (2003b).

The first three peaks and three dips in the power spectrum are detected with high confidence. Although the data favor a fourth peak in the vicinity of $l=1055$, with an amplitude of $\mathcal{C}_{1055}=$ $1020 \mu \mathrm{K}^{2}$, the marginalized likelihood for the curvature parameter is not inconsistent with zero. For comparison, the same analysis is applied to the B98 data from the Ruhl et al. (2003) release, as well as the binned first-year WMAP data (Bennett et al. 2003b). The results of all three analyses are compared in Table 3 and indicate a remarkable degree of consistency between the three independent experiments.

The degree of concordance in temperature observations is further illustrated in Figure 8, which shows a compilation of the power spectrum estimates derived from four experiments, representing four very different experimental approaches, with observations probing nearly a decade in electromagnetic frequency.

\section{CONCLUSION}

We derive an estimate of the angular power spectrum of the CMB from the data obtained with the $145 \mathrm{GHz}$ polarizationsensitive bolometers that flew on the 2003 January flight of

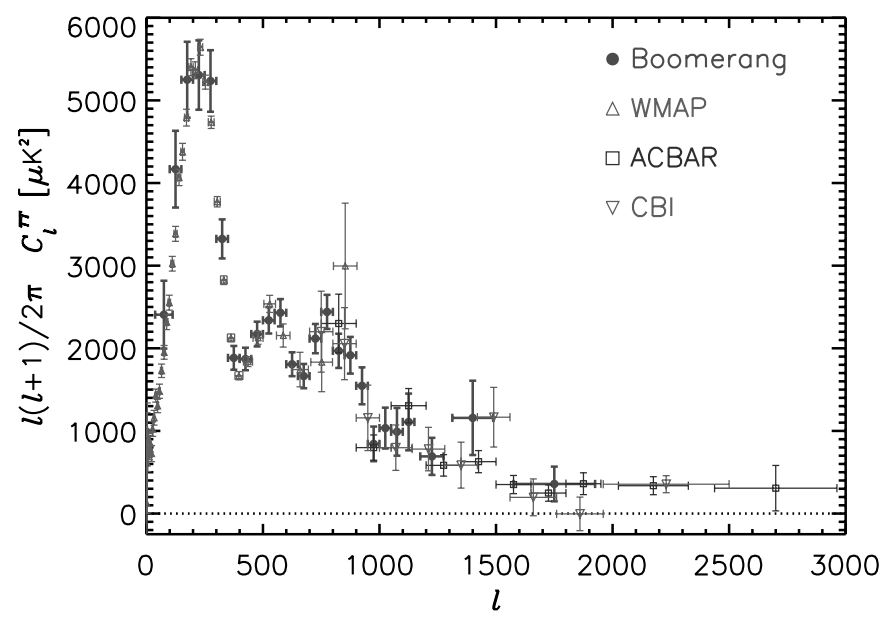

FIG. 8.-Comparison of recently published CMB power spectra, $\mathcal{C}_{l}^{\mathrm{TT}}$, (data from Hinshaw et al. [2003], Kuo et al. [2004], and Readhead et al. [2004], in addition to B03). The data shown are derived from independent measurements that span an order of magnitude in electromagnetic frequency $(20-200 \mathrm{GHz})$, employ both single-dish and interferometric instruments, and operate from terrestrial, balloon-borne, and orbital platforms. The remarkable degree of accord between such a diverse set of measurements is indicative of the maturity of the observational field. [See the electronic edition of the Journal for a color version of this figure.]
BOOMERANG. The 245 and $345 \mathrm{GHz}$ channels place stringent limits on the level of foreground contamination. We characterize the systematic effects that result from various instrumental calibration uncertainties with Monte Carlo analyses and verify the consistency of the data with jackknife tests.

The B03 data are the first to be obtained using PSBs, which are identical in design to the polarized pixels in the Planck HFI focal plane. The sensitivity per resolution element achieved in the B03 deep field is comparable to that anticipated by Planck at $143 \mathrm{GHz}$. The high signal-to-noise ratio of the temperature data results in a sample variance-limited estimate of the power spectrum at multipoles $l \lesssim 900$.

The B03 data presented in this work represent the most precise measurements to date of the angular power spectrum between $l=600$ and 1200 . In this regard, B03 plays a valuable role in bridging the gap between the all-sky WMAP survey at $l \lesssim 600$ and the high angular resolution data from terrestrial observations above $l \gtrsim 1200$. We characterize a series of features in the power spectrum that extend to multipoles $l \gtrsim 1000$, consistent with those expected from acoustic oscillations in the primordial plasma in the context of standard cosmologies.

We gratefully acknowledge support from the CIAR, CSA, and NSERC in Canada, ASI, University La Sapienza, and PNRA in Italy, PPARC and the Leverhulme Trust in the UK, and NASA (awards NAG5-9251 and NAG5-12723) and NSF (awards OPP 99-80654 and OPP 04-07592) in the US. Additional support for detector development was provided by CIT and the Jet Propulsion Laboratory (JPL). C. B. N. acknowledges support from a Sloan Foundation Fellowship; W. C. J. and T. E. M. were partially supported by NASA GSRP Fellowships. Field, logistical, and flight support were supplied by USAP and NSBF; data recovery was particularly appreciated. This research used resources at NERSC, supported by the DOE under contract DE-AC0376SF00098, and the MacKenzie cluster at CITA, funded by the Canada Foundation for Innovation. We also thank the CASPUR, Rome, computational facilities and the Applied Cluster Computing Technologies Group at JPL for computing time and technical support. Some of the results in this paper have been derived using the HEALPix package (Górski et al. 2005), and nearly all have benefitted from the FFTW implementation of discrete Fourier transforms (Frigo \& Johnson 2005). Finally, we would like to thank the referee for making significant contributions to the quality of this paper. 


\section{REFERENCES}

Bartlett, J. G., Douspis, M., Blanchard, A., \& Le Dour, M. 2000, A\&AS, 146, 507

Bennett, C. L., et al. 2003a, ApJ, 583, 2003b, ApJS, 148, 1

Bond, J. R., Jaffe, A. H., \& Knox, L. 1998, Phys. Rev. D, 57, 2117 2000, ApJ, 533, 19

Borrill, J. 1999, in AIP Conf. Proc. 476, 3K Cosmology, ed. L. Maiani, F. Melchiorri, \& N. Vittorio (Woodbury: AIP), 277

Brandt, W. N., Lawrence, C. R., Readhead, A. C. S., Pakianathan, J. N., \& Fiola, T. M. 1994, ApJ, 424, 1

Bridle, S. L., Crittenden, R., Melchiorri, A., Hobson, M. P., Kneissl, R., \& Lasenby, A. N. 2002, MNRAS, 335, 1193

Crill, B. P., et al. 2003, ApJS, 148, 527

de Bernardis, P., et al. 2000, Nature, 404, 955 -. 2002, ApJ, 564, 559

de Gasperis, G., Balbi, A., Cabella, P., Natoli, P., \& Vittorio, N. 2005, A\&A, 436, 1159

de Oliveira-Costa, A. 2005, in ASP Conf. Ser. 343, Astronomical PolarimetryCurrent Status and Future Directions, ed. A. Adamson, C. Aspin, \& C. J. Davis (San Fransisco: ASP), 485

de Oliveira-Costa, A., Tegmark, M., Gutierrez, C. M., Jones, A. W., Davies, R. D., Lasenby, A. N., Rebolo, R., \& Watson, R. A. 1999, ApJ, 527, L9

Dickinson, C., et al. 2004, MNRAS, 353, 732

Finkbeiner, D., Davis, M., \& Schlegel, D. 1999, ApJ, 524, 867

Frigo, M., \& Johnson, S. G. 2005, IEEE Proc., 93, 216

Górski, K. M., Hivon, E., Banday, A. J., Wandelt, B. D., Hansen, F. K., Reinecke, M., \& Bartelmann, M. 2005, ApJ, 622, 759

Halverson, N. W., et al. 2002, ApJ, 568, 38

Hauser, M. G., Kelsall, T., Leisawitz, D., \& Weiland, J. 1998, COBE Diffuse Infrared Background Experiment Explanatory Version 2.3 (COBE Ref. Pub. 98-A; Greenbelt:NASA/GSFC)
Hinshaw, G., et al. 2003, ApJS, 148, 135

Hivon, E., Górski, K. M., Netterfield, C. B., Crill, B. P., Prunet, S., \& Hansen, F. 2002, ApJ, 567, 2

Jones, W. C. 2005, Ph.D. thesis, Caltech

Jones, W. C., Bhatia, R. S., Bock, J. J., \& Lange, A. E. 2003, Proc. SPIE, 4855, 227

Jones, W. C., et al. 2006, A\&A, submitted (astro-ph/0606606)

Kuo, C. L., et al. 2004, ApJ, 600, 32

Lee, A. T., et al. 2001, ApJ, 561, L1

MacTavish, C., et al. 2006, ApJ, 647, 799

Masi, S., et al. 2001, ApJ, 553, L93 2006, A\&A, submitted (astro-ph/0507509)

Montroy, T. E. 2003, Ph.D. thesis, Univ. California (Santa Barbara)

Montroy, T., et al. 2003, NewA Rev., 47, 1057 . 2006, ApJ, 647, 813

Netterfield, C. B., et al. 2002, ApJ, 571, 604

Neugebauer, G., et al. 1984, ApJ, 278, L1

Page, L., et al. 2003a, ApJS, 148, 39 2003b, ApJS, 148, 233

Piacentini, F., et al. 2006, ApJ, 647, 833

Polenta, G., Marnucci, D., Balbi, A., de Bernardis, P., Hivon, E., Masi, S., Natoli, P., \& Vittorio, N. 2005, J. Cosmol. Astropart. Phys., 11

Prunet, S., et al. 2001, in Mining the Sky, ed. A. J. Banday, S. Zaroubi, \& M. Bartelmann (Berlin: Springer), 421

Readhead, A. C. S., et al. 2004, ApJ, 609, 498

Ruhl, J. E., et al. 2003, ApJ, 599, 786

Schlegel, D. J., Finkbeiner, D. P., \& Davis, M. 1998, ApJ, 500, 525

Tristram, M., Macías-Pérez, J. F., Renault, C., \& Santos, D. 2005a, MNRAS, 358,833

Tristram, M., et al. 2005b, A\&A, 436, 785 